\title{
Facilitators and Barriers to the Adoption of Telemedicine During the First Year of COVID-19: Systematic Review
}

Clemens Kruse, MBA, MHA, MSIT, PhD; Katharine Heinemann, MHA

School of Health Administration, Texas State University, San Marcos, TX, United States

Corresponding Author:

Clemens Kruse, MBA, MHA, MSIT, PhD

School of Health Administration

Texas State University

Encino Hall, Room 250

601 University Dr

San Marcos, TX, 78666

United States

Phone: 15122454462

Fax: 15122458712

Email: scottkruse@txstate.edu

\section{Abstract}

Background: The virulent and unpredictable nature of COVID-19 combined with a change in reimbursement mechanisms both forced and enabled the rapid adoption of telemedicine around the world. Thus, it is important to now assess the effects of this rapid adoption and to determine whether the barriers to such adoption are the same today as they were under prepandemic conditions.

Objective: The objective of this systematic literature review was to examine the research literature published during the COVID-19 pandemic to identify facilitators, barriers, and associated medical outcomes as a result of adopting telemedicine, and to determine if changes have occurred in the industry during this time.

Methods: The systematic review was performed in accordance with the Kruse protocol and the results are reported in accordance with the PRISMA (Preferred Reporting Items for Systematic Reviews and Meta-Analyses) guidelines. We analyzed 46 research articles from five continents published during the first year of the COVID-19 pandemic that were retrieved from searches in four research databases: PubMed (MEDLINE), CINAHL, Science Direct, and Web of Science.

Results: Reviewers identified 25 facilitator themes and observations, 12 barrier themes and observations, and 14 results (compared to a control group) themes and observations. Overall, $22 \%$ of the articles analyzed reported strong satisfaction or satisfaction (zero reported a decline in satisfaction), 27\% reported an improvement in administrative or efficiency results (as compared with a control group), 14\% reported no statistically significant difference from the control group, and $40 \%$ and $10 \%$ reported an improvement or no statistically significant difference in medical outcomes using the telemedicine modality over the control group, respectively.

Conclusions: The pandemic encouraged rapid adoption of telemedicine, which also encouraged practices to adopt the modality regardless of the challenges identified in previous research. Several barriers remain for health policymakers to address; however, health care administrators can feel confident in the modality as the evidence largely shows that it is safe, effective, and widely accepted.

(J Med Internet Res 2022;24(1):e31752) doi: 10.2196/31752

\section{KEYWORDS}

telemedicine; pandemic; technology acceptance; COVID-19; digital health; telehealth; health policy; health care 


\section{Introduction}

\section{Rationale}

The virulent nature of COVID-19 forced social distancing and a decrease of in-person visits to clinics around the world. Telemedicine presented health care providers with solutions that enabled a social-distancing window into the clinical environment and a continuation of the doctor-patient relationship.

Telemedicine is defined by the World Health Organization as healing from a distance through information communications technologies by all health care professionals for the "exchange of valid information for diagnosis, treatment and prevention of disease and injuries, research and evaluation" [1]. Telemedicine is not a perfect means of patient care; however, it offers great advantages to overcome geographical barriers to improve health outcomes [1]. Validated and peer-reviewed international statistics are elusive on adoption figures, but a recent question-and-answer session indicates overall low adoption of telemedicine internationally [2]. In the United States, prior to the pandemic, telemedicine had only been adopted by $8 \%$ of providers [3]. Providers have recognized wide acceptance of telemedicine by patients; however, prior to the desperate circumstances of COVID-19, they had not been willing to adopt telemedicine on a wide scale [4]. The largest challenges to the adoption of telemedicine were identified as technically challenged staff, resistance to change, cost, reimbursement, and education level of the patient [5]. Telemedicine saves patients time, consultation fees, and travel expenses [6]. However, telemedicine requires users at both ends to possess certain levels of technological skills such as those required to enable video teleconferencing [7]. Fortunately, some countries enacted legislation to expand the adoption of telemedicine. For example, in the United States, telemedicine was not easily reimbursed by federal programs until the Coronavirus Aid, Relief, and Economic Security (CARES) Act legislation [8], which greatly increased reimbursement mechanisms for the telemedicine modality. This change in reimbursement structure should not be ignored, and it most likely provided a significant catalyst to the adoption of telemedicine.

A large number of articles were published in the first 12 months of the pandemic (February 2020 to February 2021) on the rapid implementation efforts of telemedicine to enable clinics and hospitals to continue to see patients and care for their needs $[9,10]$. However, providers acknowledge some of the shortfalls inherent to this modality, such as lack of technical infrastructure, cost, lack of technical staff, computer literacy of both staff and patients, and a negative impact on the patient-to-provider relationship [4,11-13]. A systematic review performed in 2020 on telemedicine and COVID-19 evaluated 44 articles along four service lines and identified 10 themes of efficiency [14]. However, the authors did not evaluate facilitators and barriers to adoption or health outcomes. Another systematic review [5] was performed in 2016 on the barriers to the adoption of telemedicine worldwide, which evaluated 30 articles across all service lines in all countries; however, it also did not evaluate facilitators or health outcomes.
Although analyses have been published that highlight the advantages to the adoption of telemedicine, with an $8 \%$ adoption rate in the United States, the conclusions of these previous studies may not be as robust as possible. The circumstances presented by the pandemic have encouraged wider adoption of this modality of care. Therefore, with proper systematic review techniques, reviewer observations this far into the pandemic will undoubtedly be more robust and widely applicable to medicine.

\section{Objectives}

The purpose of this systematic review was to evaluate the facilitators and barriers to the adoption of telemedicine worldwide, including an analysis of health outcomes and patient satisfaction. A brief comparison of the results of this review with those of reviews performed prior to COVID-19 was further performed to identify changes in these factors in light of the pandemic.

\section{Methods}

\section{Protocol and Registration}

The Kruse protocol for writing a systematic review was followed, and the findings are reported in accordance with the PRISMA (Preferred Reporting Items for Systematic Reviews and Meta-Analysis) guidelines [15,16]. This systematic review was registered in PROSPERO on August 2, 2021 (ID CRD42021235933).

\section{Eligibility Criteria}

The search parameters were established to find articles published in 2020 and 2021 concerning telemedicine in all aspects of care and for all ages of patients, published in peer-reviewed journals, using any method of study (mixed method, quantitative, and qualitative). Other systematic reviews were excluded because we wanted to compare our results to these previous reviews without confounding the findings. The Johns Hopkins Nursing Evidence-Based Practice Rating Scale (JHNEBP) was used to assess the quality of all articles analyzed [17]. Any studies below level IV C were discarded due to poor quality.

\section{Information Sources}

Four research databases were searched: PubMed (MEDLINE), CINAHL (excluding MEDLINE), Web of Science, and Science Direct. We also performed a journal-specific search of the Journal of Medical Internet Research.

\section{Search Strategy}

Google Scholar was used to determine the general trends of publication on this topic previously and to collect key terms from published articles. These key terms were entered into the US Library of Medicine's Medical Subject Headings (MeSH) to create an exhaustive search string using Boolean terms. The actual search string used was: (telemedicine OR telehealth OR "mobile health" OR mhealth OR ehealth) AND (COVID-19 OR coronavirus). The same search string was used in all databases. Similar filters were used in each database (not all filters are the same between databases). 


\section{Study Selection Process}

Once the search string was entered into each database, we filtered the results and screened abstracts for applicability. Although filters for the four research databases differ, we generally filtered for the date range (2020-2021), scholarly journals (no theses or opinions), and "full text" to ensure that we would have access to the entire article. Articles were rejected for a variety of reasons: protocol (no results to analyze); opinion (no data); reviews; did not use telemedicine; or did not contribute to our objective statement of identifying facilitators, barriers, or effects on patient satisfaction. The $\kappa$ statistic was calculated to identify the level of agreement between reviewers [18].

\section{Data Collection Process}

An Excel spreadsheet was used as a data-extraction tool to collect data for reporting and analysis. This spreadsheet was standardized according to the Kruse protocol [15]. We held three consensus meetings to screen abstracts, analyze articles, and discuss possible themes. After the second consensus meeting, we performed a narrative analysis to identify themes in the articles analyzed [19]. Because there were only two authors on this project, both authors analyzed all articles $(n=46)$.

\section{Data Items}

In accordance with the Kruse protocol, PRISMA standard, and JHNEBP, the following fields were collected: database source; date of publication; journal; authors; study title; PICOS (participants, intervention, results, outcomes, study design); sample size; bias within study; effect size; country of origin; statistics used; quality metrics from the JHNEBP scale; and reviewer observations as they relate specifically to the objective statement in areas of patient satisfaction, and facilitators and barriers to adoption [15,17,20]. All data items were independently collected and discussed in subsequent consensus meetings.

\section{Risk of Bias Within and Across Studies}

The JHNEBP rating scale was used for assessment of bias within and across studies. Observations of bias and methodological weaknesses were noted [17]. The JHNEBP ratings also provided insight into bias because poor-quality results can limit the external validity of the experiment.

\section{Summary Measures}

Because we included mixed methods and qualitative studies, we were unable to standardize summary measures as would be performed in a meta-analysis.

\section{Additional Analyses}

We performed a narrative, or thematic, analysis of the observations to convert them into themes (common threads between articles) [19]. We calculated the frequency of occurrence of both themes and individual observations and report these in a series of affinity matrices (tables). This technique was used to identify the statistical probability for identifying each theme, which does not identify a level of importance but rather identifies a frequency of mention of these themes in the literature during the period of observation.

\section{Results}

\section{Study Selection}

The database search and study selection process are illustrated in Figure 1. The $\kappa$ statistic was 0.95 , indicating almost perfect agreement between reviewers [18,21]. Several studies made it through all filters, but were still eliminated because they were protocols (no results), opinions, out of the date range, or other systematic reviews. 
Figure 1. Article search and selection process. WoS: Web of Science.

Identification of studies via databases and registers

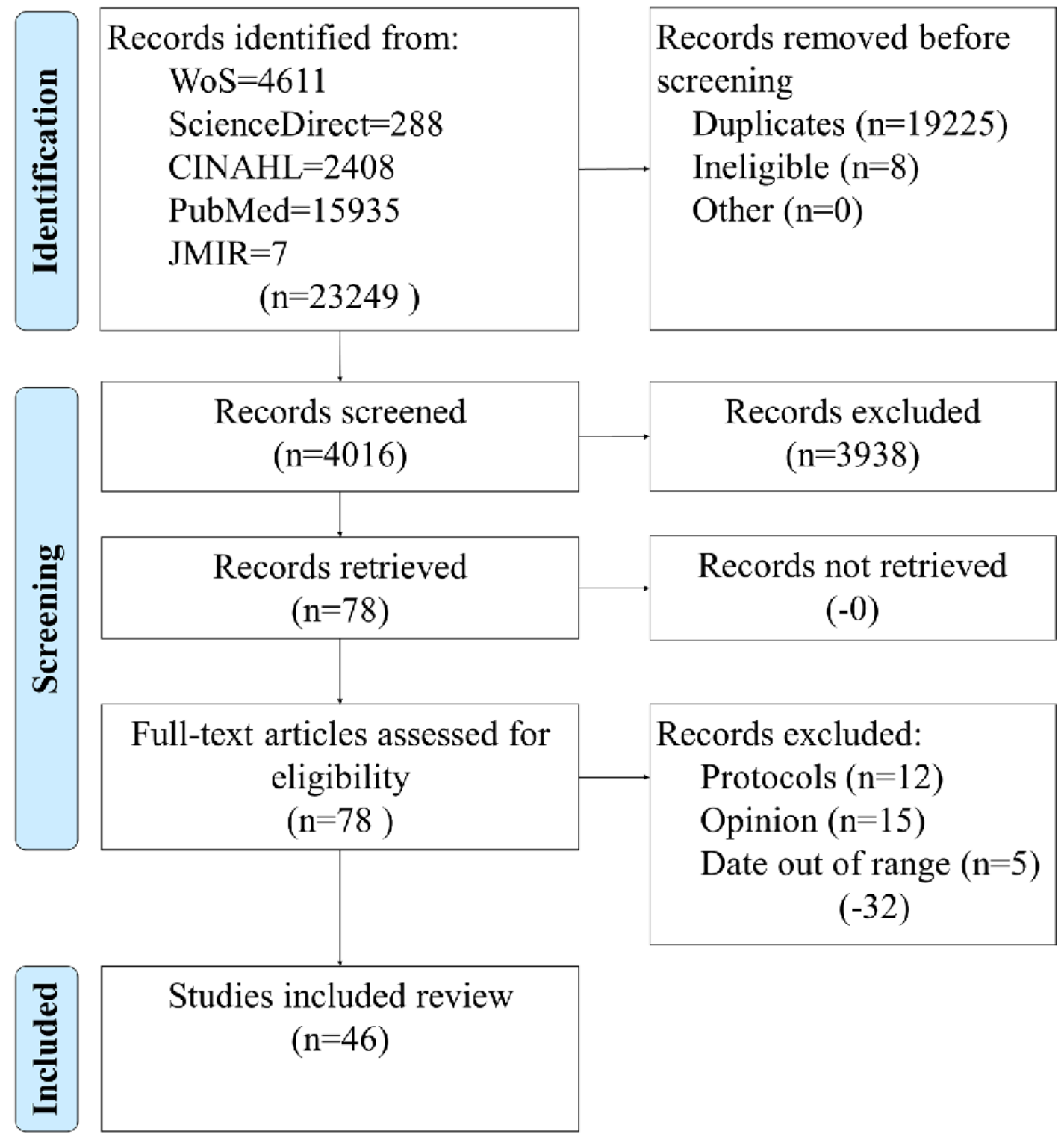

\section{Study Characteristics}

Reviewers collected study characteristics identified by the PRISMA standard such as PICOS (see Table 1). Of the 46 six studies analyzed over the 15-month period, $2(4 \%)$ involved adolescents, $6(13 \%)$ involved adults $>60$ years, and $38(83 \%)$ involved adults $>18$ years as participants. Most participants were current or former patients who agreed to participate in studies. More than half the interventions were mobile health (mHealth), telephone/televideo, or eHealth (26/46, 56\%). The rest were interventions involving telemonitoring, patient portals, telecoaching, web chat, and social media, which could be cross-platform. In these 46 studies, 18 resulted in a positive outcome over a control group (23\%), 12 of which involved medically measured outcomes $(21 \%)$ as opposed to clinical and administrative outcomes. Only 9 of the 46 (20\%) studies resulted in no statistically significant difference between the intervention and control groups, which means that positive results could be obtained through telemedicine commensurate with those obtained using traditional means of care. Four articles analyzed were published in 2021 [22-25], with the remaining 42 articles published in 2020 [26-67]. Further explanation of the results and medical outcomes can be found the Additional Analysis subsection. 
Table 1. Characteristics of the included studies according to the PICOS (Participants, Intervention, Results, Outcomes, and Study Design) structure.

\begin{tabular}{|c|c|c|c|c|c|}
\hline Study & Participant & Intervention & $\begin{array}{l}\text { Results (compared to } \\
\text { the control group or } \\
\text { other studies) }\end{array}$ & Medical outcomes & Design \\
\hline Ben-Arye et al [22] & $\begin{array}{l}\text { Adult patients ( }>18 \\
\text { years) undergoing adju- } \\
\text { vant, neoadjuvant, or } \\
\text { palliative treatment for } \\
\text { solid tumors }\end{array}$ & eHealth & $\begin{array}{l}\text { Improved compli- } \\
\text { ance/adherence }\end{array}$ & Not reported & $\begin{array}{l}\text { Prospective, controlled, } \\
\text { and nonrandomized } \\
\text { study }\end{array}$ \\
\hline Yu et al [25] & $\begin{array}{l}\text { Older adult patients } \\
(50 \%>60 \text { years, } 60 \% \\
\text { women, } 68 \% \text { one-time } \\
\text { telehealth users) and } 45 \\
\text { physicians }\end{array}$ & Telephone or televideo & $\begin{array}{l}\text { Improved patient satis- } \\
\text { faction }\end{array}$ & Not reported & Cross-sectional \\
\hline Richards et al [24] & $\begin{array}{l}\text { Adult respondents from } \\
\text { a neurosurgical outpa- } \\
\text { tient clinic (mean age } \\
63 \text { years, } 50.3 \% \text { men) }\end{array}$ & Telephone or televideo & $\begin{array}{l}\text { Improved patient satis- } \\
\text { faction }\end{array}$ & Not reported & Qualitative \\
\hline Kurihara et al [23] & $\begin{array}{l}\text { Adult patients with } \\
\text { Parkinson disease ( } 61 \% \\
\text { women, mean age } 67 \\
\text { years) at Fukuoka Uni- } \\
\text { versity Hospital }\end{array}$ & $\begin{array}{l}\text { Telemedicine self-test- } \\
\text { ing }\end{array}$ & $\begin{array}{l}\text { No control group (non- } \\
\text { experimental) }\end{array}$ & Not reported & Cross-sectional \\
\hline Alkire et al [26] & $\begin{array}{l}\text { Adults (Gen X, Millen- } \\
\text { nial) }\end{array}$ & Patient portals & $\begin{array}{l}\text { No control group (non- } \\
\text { experimental) }\end{array}$ & Not reported & Nonexperimental \\
\hline Ballin et al [27] & $\begin{array}{l}\text { Older adults, } 70 \text {-year- } \\
\text { old men, and women } \\
\text { with central obesity }\end{array}$ & $\begin{array}{l}\text { Supervised and web- } \\
\text { based }\end{array}$ & $\begin{array}{l}\text { No significant differ- } \\
\text { ence; decreased fat } \\
\text { mass }\end{array}$ & $\begin{array}{l}\text { Improved in at least } \\
\text { one area: decreased } \\
\text { fat mass }\end{array}$ & $\begin{array}{l}\text { Randomized controlled } \\
\text { trial }\end{array}$ \\
\hline Banbury et al [28] & $\begin{array}{l}\text { Adults }>50 \text { years with } \\
\text { at least one chronic } \\
\text { condition }\end{array}$ & Telemonitoring & $\begin{array}{l}\text { Telemedicine improved } \\
\text { results compared to } \\
\text { control: companionship, } \\
\text { emotional support, } \\
\text { health literacy, self- } \\
\text { management }\end{array}$ & Not reported & $\begin{array}{l}\text { Mixed methods, quasi- } \\
\text { experimental, nonran- } \\
\text { domized trial }\end{array}$ \\
\hline Barnett et al [29] & $\begin{array}{l}\text { Adults ( } 22-27 \text { years; } 10 \\
\text { men, } 10 \text { women), } \\
\text { clients of an alcohol } \\
\text { and drug counseling } \\
\text { service across Aus- } \\
\text { tralia, and } 8 \text { counselors }\end{array}$ & Webchat & $\begin{array}{l}\text { No control group (non- } \\
\text { experimental) }\end{array}$ & Not reported & $\begin{array}{l}\text { Qualitative study, non- } \\
\text { experimental }\end{array}$ \\
\hline Batalik et al [30] & $\begin{array}{l}\text { Adult cardiac rehabilita- } \\
\text { tion patients }\end{array}$ & Home-based telerehab & $\begin{array}{l}\text { No statistically signifi- } \\
\text { cant difference }\end{array}$ & $\begin{array}{l}\text { No statistically sig- } \\
\text { nificant difference }\end{array}$ & $\begin{array}{l}\text { Randomized controlled } \\
\text { trial }\end{array}$ \\
\hline Beller et al [31] & $\begin{array}{l}\text { Adult patients sched- } \\
\text { uled for video visits } \\
\text { through the University } \\
\text { of Virginal urology de- } \\
\text { partments }\end{array}$ & Televideo & $\begin{array}{l}\text { No control group (non- } \\
\text { experimental) }\end{array}$ & Not reported & Cohort \\
\hline Bernabe-Ortiz et al [32] & $\begin{array}{l}\text { Adult participants from } \\
\text { a randomized clinical } \\
\text { trial on a 1-year } \\
\text { mHealth }^{\text {a }} \text { intervention } \\
\text { on blood pressure and } \\
\text { body weight } 4 \text { years } \\
\text { postcompletion }\end{array}$ & mHealth & $\begin{array}{l}\text { Telemedicine improved } \\
\text { results compared to } \\
\text { control: decreased fat } \\
\text { mass }\end{array}$ & $\begin{array}{l}\text { Improved in at least } \\
\text { one area; decreased } \\
\text { body weight }\end{array}$ & $\begin{array}{l}\text { Retrospective study of } \\
\text { a randomized clinical } \\
\text { trial }\end{array}$ \\
\hline Bilgrami et al [33] & $\begin{array}{l}\text { Adults with inflammato- } \\
\text { ry bowel disease }\end{array}$ & $\begin{array}{l}\text { Telemedicine self-test- } \\
\text { ing }\end{array}$ & $\begin{array}{l}\text { No statistically signifi- } \\
\text { cant difference }\end{array}$ & $\begin{array}{l}\text { No statistically sig- } \\
\text { nificant difference }\end{array}$ & $\begin{array}{l}\text { Randomized controlled } \\
\text { trial }\end{array}$ \\
\hline Broers et al [34] & $\begin{array}{l}\text { Adult patients with car- } \\
\text { diovascular disease }\end{array}$ & eHealth & $\begin{array}{l}\text { No statistically signifi- } \\
\text { cant difference; in- } \\
\text { creased quality of life }\end{array}$ & Not reported & $\begin{array}{l}\text { Randomized controlled } \\
\text { trial }\end{array}$ \\
\hline
\end{tabular}




\begin{tabular}{|c|c|c|c|c|c|}
\hline Study & Participant & Intervention & $\begin{array}{l}\text { Results (compared to } \\
\text { the control group or } \\
\text { other studies) }\end{array}$ & Medical outcomes & Design \\
\hline Cho et al [35] & $\begin{array}{l}\text { Adult participants (30- } \\
59 \text { years) with at least } \\
2 \text { conditions defined by } \\
\text { the Third Report of the } \\
\text { National Cholesterol } \\
\text { Education Program ex- } \\
\text { pert panel (abdominal } \\
\text { obesity, high blood } \\
\text { pressure, high triglyc- } \\
\text { erides, low high-density } \\
\text { lipoprotein cholesterol, } \\
\text { and high fasting glu- } \\
\text { cose level) }\end{array}$ & mHealth & $\begin{array}{l}\text { Telemedicine improved } \\
\text { results compared to } \\
\text { control (decreased fat } \\
\text { mass) }\end{array}$ & $\begin{array}{l}\text { Improved in at least } \\
\text { one area: decreased } \\
\text { fat mass, decreased } \\
\text { body weight }\end{array}$ & $\begin{array}{l}\text { Randomized controlled } \\
\text { trial }\end{array}$ \\
\hline Claes et al [36] & $\begin{array}{l}\text { Adult patients with car- } \\
\text { diovascular disease } \\
\text { from } 3 \text { European hospi- } \\
\text { tals }\end{array}$ & eHealth & $\begin{array}{l}\text { Improved health behav- } \\
\text { iors }\end{array}$ & Not reported & $\begin{array}{l}\text { Randomized controlled } \\
\text { trial }\end{array}$ \\
\hline Coorey et al [37] & $\begin{array}{l}\text { Adults who had com- } \\
\text { pleted } 12 \text { months of } \\
\text { follow-up from the } \\
\text { Consumer Navigation } \\
\text { of Electronic Cardiovas- } \\
\text { cular Tools trial }\end{array}$ & eHealth & $\begin{array}{l}\text { No control group (non- } \\
\text { experimental): im- } \\
\text { proved self-manage- } \\
\text { ment, improved health } \\
\text { literacy }\end{array}$ & Not reported & $\begin{array}{l}\text { Qualitative analysis of } \\
\text { a randomized controlled } \\
\text { study }\end{array}$ \\
\hline Ding et al [38] & $\begin{array}{l}\text { Adults (mean age } 70.1 \\
\text { years) with chronic } \\
\text { heart failure }\end{array}$ & Telemonitoring & $\begin{array}{l}\text { Telemedicine improved } \\
\text { results compared to } \\
\text { controls: improved } \\
\text { compliance/adherence }\end{array}$ & Not reported & $\begin{array}{l}\text { Randomized controlled } \\
\text { trial }\end{array}$ \\
\hline Geramita et al [39] & $\begin{array}{l}\text { Adult lung transplant } \\
\text { recipients }\end{array}$ & mHealth & $\begin{array}{l}\text { No statistically signifi- } \\
\text { cant difference }\end{array}$ & Not reported & $\begin{array}{l}\text { Randomized controlled } \\
\text { follow-up study }\end{array}$ \\
\hline Gong et al [40] & Adult hypertension & mHealth & $\begin{array}{l}\text { Telemedicine improved } \\
\text { results compared to } \\
\text { controls: improved } \\
\text { compliance/adherence }\end{array}$ & $\begin{array}{l}\text { Improved in at least } \\
\text { one area: reductions } \\
\text { in blood pressure }\end{array}$ & $\begin{array}{l}\text { Randomized controlled } \\
\text { trial }\end{array}$ \\
\hline Han et al [41] & $\begin{array}{l}\text { Adults (<55 years) } \\
\text { prepandemic (S1) and } \\
273 \text { follow-up surveys } \\
\text { (S2); university-affiliat- } \\
\text { ed, and physicians }\end{array}$ & eHealth & $\begin{array}{l}\text { No control group (non- } \\
\text { experimental): } \\
\text { telemedicine improved } \\
\text { results compared to } \\
\text { controls, improved } \\
\text { compliance/adherence }\end{array}$ & Not reported & Qualitative \\
\hline Harding et al [42] & $\begin{array}{l}\text { Adult caregivers with } \\
837 \text { patient assessment } \\
\text { outcomes }\end{array}$ & mHealth & $\begin{array}{l}\text { No control group (non- } \\
\text { experimental) }\end{array}$ & Not reported & Qualitative (pilot study) \\
\hline Hsia et al [43] & $\begin{array}{l}\text { Pediatric patients with } \\
\text { asthma }\end{array}$ & mHealth & $\begin{array}{l}\text { Telemedicine improved } \\
\text { results compared to } \\
\text { controls: improved self- } \\
\text { management, improved } \\
\text { patient satisfaction }\end{array}$ & $\begin{array}{l}\text { Improved self-man- } \\
\text { agement, decreased } \\
\text { medication use, in- } \\
\text { crease in controlled } \\
\text { asthma }\end{array}$ & Prospective study \\
\hline Hsieh et al [44] & $\begin{array}{l}\text { Insured adults (>20 } \\
\text { years) }\end{array}$ & Patient portals & $\begin{array}{l}\text { No control group (non- } \\
\text { experimental) }\end{array}$ & Not reported & Qualitative \\
\hline Hutchesson et al [45] & $\begin{array}{l}\text { Adult Australian wom- } \\
\text { en with a recent history } \\
\text { of preeclampsia }\end{array}$ & mHealth & $\begin{array}{l}\text { No statistically signifi- } \\
\text { cant difference }\end{array}$ & $\begin{array}{l}\text { No statistically sig- } \\
\text { nificant difference }\end{array}$ & $\begin{array}{l}\text { Pilot randomized con- } \\
\text { trolled trial }\end{array}$ \\
\hline $\begin{array}{l}\text { Jiménez-Marrero et al } \\
\text { [46] }\end{array}$ & $\begin{array}{l}\text { Adult patients with } \\
\text { chronic heart failure }\end{array}$ & Televideo & $\begin{array}{l}\text { Telemedicine improved } \\
\text { results compared to } \\
\text { controls, decreased cost }\end{array}$ & $\begin{array}{l}\text { Improved in at least } \\
\text { one area: decreased } \\
\text { incidence of heart } \\
\text { failure }\end{array}$ & $\begin{array}{l}\text { Randomized controlled } \\
\text { trial }\end{array}$ \\
\hline
\end{tabular}




\begin{tabular}{|c|c|c|c|c|c|}
\hline Study & Participant & Intervention & $\begin{array}{l}\text { Results (compared to } \\
\text { the control group or } \\
\text { other studies) }\end{array}$ & Medical outcomes & Design \\
\hline Katt et al [47] & $\begin{array}{l}180 \text { patients with up- } \\
\text { per-extremity condition } \\
\text { and } 302 \text { physicians }\end{array}$ & Telephone or televideo & $\begin{array}{l}\text { Improved patient satis- } \\
\text { faction }\end{array}$ & Not reported & Qualitative \\
\hline Kobe et al [48] & $\begin{array}{l}\text { Adult patients ( } 52 \% \\
\text { men, mean age } 62 \\
\text { years, } 55.5 \% \text { African } \\
\text { American) of Duke } \\
\text { University Health Sys- } \\
\text { tem with type } 2 \text { dia- } \\
\text { betes, poorly controlled } \\
\text { hypertension, and on } \\
\text { prescription hyperten- } \\
\text { sion and diabetes medi- } \\
\text { cation }\end{array}$ & Telephone or televideo & $\begin{array}{l}\text { Telemedicine improved } \\
\text { results compared to } \\
\text { control }\end{array}$ & $\begin{array}{l}\text { Improved in at least } \\
\text { one area, improved } \\
\text { annual rate } \mathrm{eGFR}^{\mathrm{b}} \\
\text { decline }\end{array}$ & $\begin{array}{l}\text { Secondary analysis of } \\
\text { randomized controlled } \\
\text { trial }\end{array}$ \\
\hline Lai et al [49] & $\begin{array}{l}\text { Adults with Parkinson } \\
\text { disease (telehealth } \\
\text { mean age } 63 \text { years, } \\
\text { control mean age } 70 \\
\text { years; } 70 \% \text { men, pre- } \\
\text { dominantly White) }\end{array}$ & Telemonitoring & $\begin{array}{l}\text { Telemedicine improved } \\
\text { results compared to } \\
\text { control: improved com- } \\
\text { pliance/adherence, } \\
\text { health behaviors, and } \\
\text { patient satisfaction }\end{array}$ & Not reported & Mixed methods \\
\hline Lemelin et al [50] & $\begin{array}{l}\text { Adult women (mean } \\
\text { age } 32 \text { years) with ges- } \\
\text { tational diabetes melli- } \\
\text { tus }\end{array}$ & Telecoaching & $\begin{array}{l}\text { Improved patient satis- } \\
\text { faction: telemedicine } \\
\text { improved results com- } \\
\text { pared to control }\end{array}$ & $\begin{array}{l}\text { Identified other ar- } \\
\text { eas for intervention }\end{array}$ & $\begin{array}{l}\text { Prospective and con- } \\
\text { trolled clinical trial }\end{array}$ \\
\hline Manning et al [51] & $\begin{array}{l}\text { Adults from families } \\
\text { with toddlers }\end{array}$ & Televideo & $\begin{array}{l}\text { No statistically signifi- } \\
\text { cant difference }\end{array}$ & Not reported & $\begin{array}{l}\text { Mixed method quasiex- } \\
\text { perimental and longitu- } \\
\text { dinal design }\end{array}$ \\
\hline Martins et al [53] & $\begin{array}{l}\text { Adult patients (mean } \\
\text { age } 62 \text { years, } 50 \% \\
\text { women) with suspected } \\
\text { acute strokes at a Brazil } \\
\text { university hospital }\end{array}$ & mHealth & $\begin{array}{l}\text { Telemedicine improved } \\
\text { results compared to } \\
\text { control }\end{array}$ & $\begin{array}{l}\text { Improved in at least } \\
\text { one area: decreased } \\
\text { mortality, decreased } \\
\text { intracranial hemor- } \\
\text { rhage }\end{array}$ & $\begin{array}{l}\text { Prospective observation- } \\
\text { al }\end{array}$ \\
\hline McGillicuddy et al [54] & $\begin{array}{l}\text { Adults (mean 51.5-52.1 } \\
\text { years) with kidney } \\
\text { transplants (majority } \\
\text { men, African Ameri- } \\
\text { can) }\end{array}$ & mHealth & $\begin{array}{l}\text { Telemedicine improved } \\
\text { results compared to } \\
\text { control }\end{array}$ & $\begin{array}{l}\text { Improved in at least } \\
\text { one area: reduction } \\
\text { in mean tacrolimus } \\
\text { trough coefficient of } \\
\text { variation }\end{array}$ & $\begin{array}{l}\text { Randomized controlled } \\
\text { clinical trial }\end{array}$ \\
\hline Mo et al [55] & $\begin{array}{l}\text { Adult patients ( } 51.7- \\
53.5 \text { years) with chronic } \\
\text { heart failure (approxi- } \\
\text { mately } 66 \% \text { men) }\end{array}$ & Telephone or televideo & $\begin{array}{l}\text { Telemedicine improved } \\
\text { results compared to } \\
\text { control: improved emo- } \\
\text { tional support }\end{array}$ & $\begin{array}{l}\text { Improved in at least } \\
\text { one area: mental } \\
\text { health inventory, } \\
\text { quality of life }\end{array}$ & $\begin{array}{l}\text { Open-label intervention- } \\
\text { al study }\end{array}$ \\
\hline Mustonen et al [56] & $\begin{array}{l}\text { Adult patients ( }>45 \\
\text { years; mean age } 65 \\
\text { years) with type } 2 \text { dia- } \\
\text { betes and coronary } \\
\text { artery disease (approxi- } \\
\text { mately } 40 \% \text { women) }\end{array}$ & Telecoaching & $\begin{array}{l}\text { No statistically signifi- } \\
\text { cant difference }\end{array}$ & Not reported & $\begin{array}{l}\text { Posttrial analysis of a } \\
\text { randomized controlled } \\
\text { trial }\end{array}$ \\
\hline O'Shea et al [57] & $\begin{array}{l}\text { Adults }(77 \% \text { men, mean } \\
\text { age } 61 \text { years) }\end{array}$ & eHealth & Not reported & Not reported & $\begin{array}{l}\text { Posttrial analysis of an } \\
\text { acceptability and feasi- } \\
\text { bility trial }\end{array}$ \\
\hline Perri et al [58] & $\begin{array}{l}\text { Adults (mean } 55.4 \\
\text { years) from } 14 \text { counties } \\
\text { in Florida ( } 83 \% \text { wom- } \\
\text { en, } 73.9 \% \text { White) }\end{array}$ & Telephone or televideo & $\begin{array}{l}\text { Telemedicine improved } \\
\text { results compared to } \\
\text { control: decreased fat } \\
\text { mass, improved self- } \\
\text { management }\end{array}$ & $\begin{array}{l}\text { Improved in at least } \\
\text { one area: decreased } \\
\text { body weight }\end{array}$ & $\begin{array}{l}\text { Randomized clinical } \\
\text { trial }\end{array}$ \\
\hline
\end{tabular}




\begin{tabular}{|c|c|c|c|c|c|}
\hline Study & Participant & Intervention & $\begin{array}{l}\text { Results (compared to } \\
\text { the control group or } \\
\text { other studies) }\end{array}$ & Medical outcomes & Design \\
\hline Piera-Jiménez et al [59] & $\begin{array}{l}\text { Adults (majority 50-70 } \\
\text { years and men) from } \\
\text { Spain, the Netherlands, } \\
\text { and Taiwan }\end{array}$ & Telemonitoring & $\begin{array}{l}\text { Telemedicine improved } \\
\text { results compared to } \\
\text { control }\end{array}$ & $\begin{array}{l}\text { Improved in at least } \\
\text { one area, improved } \\
\text { quality of life }\end{array}$ & $\begin{array}{l}\text { Financial randomized } \\
\text { controlled trial }\end{array}$ \\
\hline Press et al [60] & $\begin{array}{l}\text { Adults (mean } 54.5 \\
\text { years) with asthma or } \\
\text { chronic obstructive pul- } \\
\text { monary disease (majori- } \\
\text { ty Black women) }\end{array}$ & mHealth & $\begin{array}{l}\text { Telemedicine improved } \\
\text { results compared to } \\
\text { control: improved self- } \\
\text { management health be- } \\
\text { haviors }\end{array}$ & $\begin{array}{l}\text { Increase in con- } \\
\text { trolled asthma }\end{array}$ & $\begin{array}{l}\text { Randomized controlled } \\
\text { trial }\end{array}$ \\
\hline $\begin{array}{l}\text { Ramirez-Correa et al } \\
\text { [61] }\end{array}$ & $\begin{array}{l}\text { Adults (mean } 39.9 \\
\text { years, } 56 \% \text { men) }\end{array}$ & $\begin{array}{l}\text { Telemedicine self-test- } \\
\text { ing }\end{array}$ & $\begin{array}{l}\text { No control group (non- } \\
\text { experimental) }\end{array}$ & Not reported & Cross-sectional \\
\hline Ronan et al [62] & $\begin{array}{l}\text { Adults with cystic fibro- } \\
\text { sis involved in a study } \\
\text { on an online Tai Chi in- } \\
\text { tervention }\end{array}$ & Televideo & $\begin{array}{l}\text { No statistically signifi- } \\
\text { cant difference, im- } \\
\text { proved health behaviors }\end{array}$ & Not reported & $\begin{array}{l}\text { Qualitative analysis of } \\
\text { a mixed methods ran- } \\
\text { domized controlled fea- } \\
\text { sibility study }\end{array}$ \\
\hline Sacco et al [63] & $\begin{array}{l}\text { Older adults (mean age } \\
88.2 \text { years), } 59.8 \% \\
\text { women }\end{array}$ & Telephone or video & $\begin{array}{l}\text { Improved patient satis- } \\
\text { faction, improved emo- } \\
\text { tion support }\end{array}$ & Not reported & Cross-sectional survey \\
\hline Scheerman et al [64] & $\begin{array}{l}\text { Adolescents (12-17 } \\
\text { years) and mothers }\end{array}$ & Social media & $\begin{array}{l}\text { Telemedicine improved } \\
\text { results compared to } \\
\text { control, improved } \\
\text { health behaviors }\end{array}$ & Not reported & $\begin{array}{l}\text { Cluster randomized } \\
\text { controlled trial }\end{array}$ \\
\hline Schrauben et al [65] & $\begin{array}{l}\text { Adult Chronic Renal } \\
\text { Insufficiency Cohort } \\
\text { (CRIC) Study partici- } \\
\text { pants (mean age } 68 \\
\text { years, eGFR } 54 \\
\text { mL/min } 1.73,59 \% \\
\text { men) }\end{array}$ & mHealth & $\begin{array}{l}\text { No control group (non- } \\
\text { experimental) }\end{array}$ & Not reported & Cross-sectional survey \\
\hline Shareef et al [66] & $\begin{array}{l}\text { Elderly and disabled } \\
\text { people (average age } \\
74.5 \text { years, } 59 \% \text { wom- } \\
\text { en) in retirement homes } \\
\text { and rehabilitation cen- } \\
\text { ters }\end{array}$ & $\begin{array}{l}\text { Robotics or artificial } \\
\text { intelligence }\end{array}$ & $\begin{array}{l}\text { Improved companion- } \\
\text { ship }\end{array}$ & Not reported & $\begin{array}{l}\text { Experiment and follow- } \\
\text { up survey }\end{array}$ \\
\hline van Dijk et al [67] & $\begin{array}{l}\text { Adult women (mean } \\
\text { age } 30 \text { years), either } \\
\text { less than } 13 \text { weeks } \\
\text { pregnant or trying to } \\
\text { become pregnant, and } \\
36 \text { men }\end{array}$ & mHealth & $\begin{array}{l}\text { Improved compli- } \\
\text { ance/adherence, im- } \\
\text { proved health behaviors }\end{array}$ & $\begin{array}{l}\text { Improved in at least } \\
\text { one area, improved } \\
\text { self-management }\end{array}$ & $\begin{array}{l}\text { Randomized controlled } \\
\text { trial }\end{array}$ \\
\hline
\end{tabular}

amHealth: mobile health.

b eGFR: estimated glomerular filtration rate.

\section{Risk of Bias Within and Across Studies}

Table 2 summarizes the quality indicators assessed for each article with the JHNEBP tool. The strength of evidence most frequently observed was level III followed by level I and level II. Nearly half of the articles reported strong-evidence studies that included both a control group and randomization; the next most common study type was nonexperimental (no control group) or qualitative, with the least frequent type being quasiexperimental (included a control group but no randomization). The quality of evidence most frequently observed was A (high quality), followed by B (good quality). The most common combination of strength and quality was III $\mathrm{B}$, followed closely by I A, which speaks to both the strength and quality of evidence evaluated by this review. The III B combination highlights the number of qualitative studies with smaller samples or selection bias. 
Table 2. Summary of quality assessments $(\mathrm{N}=46)$.

\begin{tabular}{lc}
\hline Evidence & Occurrence, $\mathrm{n}(\%)$ \\
\hline Strength & $22(48)$ \\
$\quad$ I (Experimental study or randomized controlled trial) & $17(37)$ \\
III (Nonexperimental, qualitative) & $7(15)$ \\
II (quasiexperimental) & $27(59)$ \\
Quality & $17(37)$ \\
A (High quality) & $2(4)$ \\
B (Good quality) &
\end{tabular}

Many studies used geographically localized samples, which may limit the external validity of the results. Some studies focused only on one gender or race, speaking to the convenience sample or volunteer-basis of their design. Asking for volunteers in a technology-oriented experiment invites bias because the self-selection allows for those who are already technology-oriented or comfortable with technology to participate. This group as the intervention can skew the results because those already comfortable with technology will not experience the frustration experienced by those who are not comfortable with technology. This selection bias also limits the external validity of the results. A comprehensive list of bias, country of origin, sample size, strength, and quality of evidence identified for each study can be found in Multimedia Appendix 1.

\section{Thematic Analysis Based on Results of Individual Studies}

During the analysis phase of the systematic review process, the reviewers recorded observations to identify instances of patient satisfaction, as well as both facilitators and barriers to the adoption of telemedicine. A thematic analysis was then performed to make sense of the observations [19]. Multiple instances of the same observation become a theme. A translation of observations to themes is provided in Multimedia Appendix 2. The summary of analysis is provided in Table 3, which lists the themes/observations from reviewers that correspond with the objective statement and sorts articles from the most recent to the oldest. 
Table 3. Summary of thematic analysis for individual studies.

\begin{tabular}{|c|c|c|c|}
\hline Authors & Patient satisfaction & Facilitators & Barriers \\
\hline Ben-Arye et al [22] & Not reported & $\begin{array}{l}\text { Technical literacy, availability of technology, past ex- } \\
\text { perience with technology }\end{array}$ & $\begin{array}{l}\text { Availability of technology, confidentiality/se- } \\
\text { curity }\end{array}$ \\
\hline Yu et al [25] & Strong satisfaction & $\begin{array}{l}\text { Concerns adequately addressed, improved health be- } \\
\text { haviors, pandemic created acceptance of technology }\end{array}$ & $\begin{array}{l}\text { Some patients prefer in-person consultations, } \\
\text { decrease in patient-provider communication, } \\
\text { technical literacy }\end{array}$ \\
\hline Richards et al [24] & Strong satisfaction & $\begin{array}{l}\text { Convenience of telemedicine, increased patient- } \\
\text { provider communication, concerns adequately ad- } \\
\text { dressed, increased access }\end{array}$ & Not reported \\
\hline Kurihara et al [23] & Not reported & $\begin{array}{l}\text { Pandemic created acceptance of technology, past ex- } \\
\text { perience with technology }\end{array}$ & $\begin{array}{l}\text { Some patients prefer in-person consultations, } \\
\text { technical literacy }\end{array}$ \\
\hline Alkirie et al [26] & Not reported & $\begin{array}{l}\text { Technical literacy, past experience with technology, } \\
\text { perceived usefulness, increased patient-provider com- } \\
\text { munication, perceived ease of use }\end{array}$ & $\begin{array}{l}\text { Technology needs further development, } \\
\text { technical literacy }\end{array}$ \\
\hline Ballin et al [27] & Not reported & $\begin{array}{l}\text { Increased connectedness, self-management, flexibility, } \\
\text { and access }\end{array}$ & Technology needs further development \\
\hline Banbury et al [28] & Not reported & $\begin{array}{l}\text { Enabled social interaction; decreased anxiety; in- } \\
\text { creased connectedness, technical literacy, and access; } \\
\text { televideo enables reading of body language; education; } \\
\text { convenience of telemedicine }\end{array}$ & $\begin{array}{l}\text { Health literacy, availability of technology, } \\
\text { technical literacy }\end{array}$ \\
\hline Barnett et al [29] & Not reported & $\begin{array}{l}\text { Increased efficiency, access, and patient-provider } \\
\text { communication, and improved standard of care }\end{array}$ & $\begin{array}{l}\text { Technology needs further development, de- } \\
\text { crease in patient-provider communication, } \\
\text { technical literacy, confidentiality/security }\end{array}$ \\
\hline Batalik et al [30] & Not reported & $\begin{array}{l}\text { Technical literacy, increased self-management, in- } \\
\text { creased access, increased flexibility }\end{array}$ & $\begin{array}{l}\text { Discomfort for wearable monitors, technical } \\
\text { literacy, technology needs further develop- } \\
\text { ment }\end{array}$ \\
\hline Beller et al [31] & Not reported & $\begin{array}{l}\text { Pandemic created acceptance of technology, availabil- } \\
\text { ity of technology, fewer miles driven to appointment, } \\
\text { convenience of telemedicine, faster initiation of treat- } \\
\text { ment, decreased costs }\end{array}$ & $\begin{array}{l}\text { Limits of reimbursement for telemedicine, } \\
\text { some patients prefer in-person consultations, } \\
\text { connectivity, technical literacy }\end{array}$ \\
\hline $\begin{array}{l}\text { Bernabe-Ortiz et al } \\
\text { [32] }\end{array}$ & Not reported & $\begin{array}{l}\text { Increased connectedness, increased adherence, im- } \\
\text { proved health behaviors }\end{array}$ & $\begin{array}{l}\text { Perceived lack of usefulness, lack of personal } \\
\text { desire to get better, some patients prefer in- } \\
\text { person consultations }\end{array}$ \\
\hline Bilgrami et al [33] & Not reported & Pandemic created acceptance of technology & Not reported \\
\hline Broers et al [34] & Strong satisfaction & $\begin{array}{l}\text { Perceived usefulness, perceived ease of use, increased } \\
\text { adherence }\end{array}$ & Decrease in quality of life after intervention \\
\hline Cho et al [35] & Not reported & $\begin{array}{l}\text { Increased adherence, increased self-management, in- } \\
\text { creased weight loss, technical literacy }\end{array}$ & Technical literacy, availability of technology \\
\hline Claes et al [36] & Not reported & Technical literacy, perceived ease of use & Technology needs further development \\
\hline Coorey et al [37] & Not reported & Increased adherence, increased self-management & $\begin{array}{l}\text { Lack of personal desire to get better, technol- } \\
\text { ogy needs further development, technical lit- } \\
\text { eracy }\end{array}$ \\
\hline Ding et al [38] & Not reported & Increased adherence, increased self-management & Technology needs further development, cost \\
\hline Geramita et al [39] & Not reported & $\begin{array}{l}\text { Long-term use may not be required to develop good } \\
\text { habits }\end{array}$ & $\begin{array}{l}\text { Cost, confidentiality/security, technology } \\
\text { needs further development }\end{array}$ \\
\hline Gong et al [40] & Not reported & Increased adherence, increased self-management & Not reported \\
\hline Han et al [41] & Not reported & $\begin{array}{l}\text { Pandemic created acceptance of technology, increased } \\
\text { efficiency, increased self-management, increased ac- } \\
\text { cess, availability of technology }\end{array}$ & $\begin{array}{l}\text { Cost, technical literacy, interoperability, } \\
\text { availability of technology }\end{array}$ \\
\hline Harding et al [42] & Not reported & Not reported & $\begin{array}{l}\text { Connectivity, confidentiality/security, techni- } \\
\text { cal literacy }\end{array}$ \\
\hline Hsia et al [43] & Strong satisfaction & $\begin{array}{l}\text { Increased quality of life, decreased emergency room } \\
\text { visits, increased adherence, availability of technology, } \\
\text { pandemic created acceptance of technology, perceived } \\
\text { ease of use, convenience of telemedicine }\end{array}$ & $\begin{array}{l}\text { Connectivity, technical literacy, cost, avail- } \\
\text { ability of technology }\end{array}$ \\
\hline
\end{tabular}




\begin{tabular}{|c|c|c|c|}
\hline Authors & Patient satisfaction & Facilitators & Barriers \\
\hline Hsieh et al [44] & Not reported & $\begin{array}{l}\text { Health literacy, perceived usefulness, perceived ease } \\
\text { of use }\end{array}$ & $\begin{array}{l}\text { Some patients prefer in-person consultations, } \\
\text { technical literacy, cost }\end{array}$ \\
\hline Hutchesson et al [45] & Strong satisfaction & $\begin{array}{l}\text { Increased self-management, perceived usefulness, } \\
\text { perceived ease of use }\end{array}$ & $\begin{array}{l}\text { Technology needs further development, per- } \\
\text { ceived lack of usefulness }\end{array}$ \\
\hline $\begin{array}{l}\text { Jiménez-Marrero et al } \\
\text { [46] }\end{array}$ & Not reported & $\begin{array}{l}\text { Decreased costs, increased adherence, increased self- } \\
\text { management }\end{array}$ & Cost \\
\hline Katt et al [47] & Strong satisfaction & $\begin{array}{l}\text { Convenience of telemedicine, pandemic created accep- } \\
\text { tance of technology, faster initiation of treatment, } \\
\text { perceived ease of use }\end{array}$ & $\begin{array}{l}\text { Some patients prefer in-person consultations, } \\
\text { workflow issues for providers }\end{array}$ \\
\hline Kobe et al [48] & Not reported & Not reported & Some patients prefer in-person consultations \\
\hline Lai et al [49] & Strong satisfaction & $\begin{array}{l}\text { Convenience of telemedicine, increased social support, } \\
\text { increased self-management }\end{array}$ & $\begin{array}{l}\text { Technology needs further development, con- } \\
\text { nectivity, decrease in patient-provider com- } \\
\text { munication, technical literacy }\end{array}$ \\
\hline Lemelin et al [50] & Strong satisfaction & Education, increased social support & Not reported \\
\hline Manning et al [51] & Not reported & Pandemic created acceptance of technology & Connectivity, availability of technology \\
\hline Marquez et al [52] & Not reported & $\begin{array}{l}\text { Past experience with technology, decreased costs, } \\
\text { pandemic created acceptance of technology, faster } \\
\text { initiation of treatment, increased access }\end{array}$ & Some patients prefer in-person consultations \\
\hline Martins et al [53] & Not reported & $\begin{array}{l}\text { Faster initiation of treatment, availability of technolo- } \\
\text { gy, increased access }\end{array}$ & $\begin{array}{l}\text { Lack of infrastructure, limits of reimburse- } \\
\text { ment for telemedicine, connectivity, confiden- } \\
\text { tiality/security }\end{array}$ \\
\hline $\begin{array}{l}\text { McGillicuddy et al } \\
\text { [54] }\end{array}$ & Not reported & Increased social support, health literacy & Not reported \\
\hline Mo et al [55] & Not reported & Increased quality of life, increased social support & Not reported \\
\hline Mustonen et al [56] & Not reported & Decreased costs & Not reported \\
\hline O'Shea et al [57] & Satisfaction & Increased self-management & $\begin{array}{l}\text { Technical literacy, perceived lack of useful- } \\
\text { ness, technology needs further development }\end{array}$ \\
\hline Perri et al [58] & Not reported & $\begin{array}{l}\text { Increased weight loss, increased adherence, increased } \\
\text { self-management }\end{array}$ & Not reported \\
\hline $\begin{array}{l}\text { Piera-Jiménez et al } \\
\text { [59] }\end{array}$ & Not reported & Decreased costs, no significant difference in cost care & Cost \\
\hline Press et al [60] & Not reported & Decreased costs, education, increased access & Availability of technology, technical literacy \\
\hline $\begin{array}{l}\text { Ramirez-Correa et al } \\
\text { [61] }\end{array}$ & Not reported & $\begin{array}{l}\text { Increased patient-provider communication, education, } \\
\text { pandemic created acceptance of technology }\end{array}$ & Connectivity \\
\hline Ronan et al [62] & Not reported & $\begin{array}{l}\text { Convenience of telemedicine, pandemic created accep- } \\
\text { tance of technology, increased social support }\end{array}$ & $\begin{array}{l}\text { Technical literacy, technology needs further } \\
\text { development, availability of technology }\end{array}$ \\
\hline Sacco et al [63] & Strong satisfaction & Increased social support, increased connectedness & Not reported \\
\hline Scheerman et al [64] & Not reported & Increased social support, improved standard of care & Not reported \\
\hline Schrauben et al [65] & Not reported & Health literacy, education & $\begin{array}{l}\text { Technical literacy, health literacy, confiden- } \\
\text { tiality/security }\end{array}$ \\
\hline Shareef et al [66] & Not reported & Enabled social interaction, increased social support & $\begin{array}{l}\text { Confidentiality/security, technical literacy, } \\
\text { perceived lack of usefulness }\end{array}$ \\
\hline van Dijk et al [67] & Not reported & Improved health behaviors, increased adherence & Not reported \\
\hline
\end{tabular}

Patient satisfaction was reported as "strong satisfaction" or "satisfaction" in $9(20 \%)$ and $1(2 \%)$ of the 46 studies, respectively, and 36 studies did not report any measure of patient satisfaction. No studies reported a decline in patient satisfaction as a result of using telemedicine as the intervention.
Twenty-five facilitator themes and seven individual observations were identified in the literature by the two reviewers. Only two studies did not identify facilitators. Facilitator themes are listed in Table 4. 
Table 4. Facilitator themes and individual observations $(\mathrm{N}=132)$.

\begin{tabular}{|c|c|c|}
\hline Themes/observations & References & Occurrence, $\mathrm{n}(\%)$ \\
\hline Increased self-management & {$[27,30,35,37,38,40,41,45,46,49,57,58]$} & $12(9.1)$ \\
\hline Pandemic created acceptance of technology & {$[23,25,31,33,41,43,47,51,52,61,62]$} & $11(8.3)$ \\
\hline Increased adherence & {$[32,34,35,37,38,40,43,46,58,67]$} & $10(7.6)$ \\
\hline Increased access & {$[24,27-30,41,52,53,60]$} & $9(6.8)$ \\
\hline Increased social support & {$[49,50,54,55,62-64,66]$} & $8(6.1)$ \\
\hline Convenience of telemedicine & {$[24,28,31,43,47,49,62]$} & $7(5.3)$ \\
\hline Perceived ease of use & {$[26,34,36,43-45,47]$} & $7(5.3)$ \\
\hline Decreased costs & {$[31,46,52,56,59,60]$} & $6(4.5)$ \\
\hline Education & {$[28,50,60,61,65]$} & $5(3.8)$ \\
\hline Technical literacy & {$[22,26,30,35,36]$} & $5(3.8)$ \\
\hline Availability of technology & {$[22,31,41,43,53]$} & $5(3.8)$ \\
\hline Increased patient-provider communication & {$[24,26,29,61]$} & $4(3.0)$ \\
\hline Faster initiation of treatment & {$[31,47,52,53]$} & $4(3.0)$ \\
\hline Increased connectedness & {$[27,28,32,63]$} & $4(3.0)$ \\
\hline Perceived usefulness & {$[26,34,44,45]$} & $4(3.0)$ \\
\hline Past experience with technology & {$[22,23,26,52]$} & $4(3.0)$ \\
\hline Health literacy & {$[44,54,65]$} & $3(2.3)$ \\
\hline Improved health behaviors & {$[25,32,67]$} & $3(2.3)$ \\
\hline Increased efficiency & {$[29,41]$} & $2(1.5)$ \\
\hline Concerns adequately addressed & {$[24,25]$} & $2(1.5)$ \\
\hline Enabled social interaction & {$[28,66]$} & $2(1.5)$ \\
\hline Increased quality of life & {$[43,55]$} & $2(1.5)$ \\
\hline Improved standard of care & {$[29,64]$} & $2(1.5)$ \\
\hline Increased flexibility & {$[27,30]$} & $2(1.5)$ \\
\hline Increased weight loss & {$[35,58]$} & $2(1.5)$ \\
\hline Decreased anxiety & {$[28]$} & $1(0.8)$ \\
\hline Increased technical literacy & {$[28]$} & $1(0.8)$ \\
\hline Televideo enables reading of body language & [28] & $1(0.8)$ \\
\hline Fewer miles driven to appointment & {$[31]$} & $1(0.8)$ \\
\hline Long-term use may not be required to develop good habits & [39] & $1(0.8)$ \\
\hline Decreased emergency room visits & [43] & $1(0.8)$ \\
\hline No significant difference in cost of care & [59] & $1(0.8)$ \\
\hline Not reported & {$[42,48]$} & $2\left(\mathrm{~N} / \mathrm{A}^{\mathrm{a}}\right)$ \\
\hline
\end{tabular}

${ }^{\mathrm{a}} \mathrm{N} / \mathrm{A}$ : not applicable.

The most commonly identified themes were increased self-management, acceptance of the technology from the pandemic, adherence to treatment protocols, access, and social support. For the 46 articles, these themes represent $38 \%$ of all 132 occurrences. Other themes included convenience of telemedicine and perceived ease of use, decreased cost, opportunity for education, technical literacy, availability of technology, an increase in patient-provider communication, faster initiation of treatment, increased connectedness, perceived usefulness, and past experience with technology. Health literacy and improved health behaviors were identified less frequently, and increased office efficiencies, medical concerns adequately addressed, enabled social interaction, increased quality of life, improved standard of care, increased flexibility, and increased weight loss were the least frequent themes identified. The following seven individual observations accounted for $5 \%$ of the total observations: decreased anxiety, increased technical literacy, televideo enabled reading of body language, fewer miles driven to appointment, long-term use may not be required 
to develop good habits, decreased emergency room visits, and no significant difference in cost of care.

Twelve themes and five individual observations were identified as barriers from the literature by the reviewers; 11 studies did not identify barriers (11\%). Table 5 lists the themes and individual observations.

The most commonly listed barriers were technical literacy, technology needs further development, availability of technology, and patient preference, accounting for $55 \%$ of the total 86 occurrences. Cost, connectivity, and confidentiality/security were also identified, as well as health literacy, limits of reimbursement for telemedicine, and lack of personal desire to get better with less frequent occurrences (2 each). The remaining five observations made up a total of $6 \%$ of the total occurrences: decrease in quality of life after intervention, discomfort for wearing monitors, workflow issues for providers, lack of data infrastructure, and interoperability.

Table 5. Barrier themes and individual observations $(\mathrm{N}=86)$.

\begin{tabular}{|c|c|c|}
\hline Themes/observations & References & Occurrence, $\mathrm{n}(\%)$ \\
\hline Technical literacy & {$[23,25,26,28-31,35,37,41-44,49,57,60,62,65,66]$} & $19(22)$ \\
\hline Technology needs further development & {$[26,27,29,30,36-39,45,49,57,62]$} & $12(14)$ \\
\hline Availability of technology & {$[22,28,35,41,43,51,60,62]$} & $8(9)$ \\
\hline Cost & {$[38,39,41,43,44,46,59]$} & $7(8)$ \\
\hline Connectivity & {$[31,42,43,49,51,53,61]$} & $7(8)$ \\
\hline Confidentiality/security & {$[22,29,39,42,53,65,66]$} & $7(8)$ \\
\hline Some patients prefer in-person consultations & {$[23,25,31,32,44,47,48,52]$} & $8(9)$ \\
\hline Perceived lack of usefulness & {$[32,45,57,66]$} & $4(5)$ \\
\hline Decrease in patient-provider communication & {$[25,29,49]$} & $3(3)$ \\
\hline Health literacy & {$[28,65]$} & $2(2)$ \\
\hline Limits of reimbursement for telemedicine & {$[31,53]$} & $2(2)$ \\
\hline Lack of personal desire to get better & {$[32,37]$} & $2(2)$ \\
\hline Decrease in quality of life after intervention & {$[34]$} & $1(1)$ \\
\hline Discomfort for wearable monitors & {$[30]$} & $1(1)$ \\
\hline Workflow issues for providers & [47] & $1(1)$ \\
\hline Lack of infrastructure & [53] & $1(1)$ \\
\hline Interoperability & [41] & $1(1)$ \\
\hline Not reported & {$[24,33,40,50,54-56,58,63,64,67]$} & $11\left(\mathrm{~N} / \mathrm{A}^{\mathrm{a}}\right)$ \\
\hline
\end{tabular}

${ }^{\mathrm{a}} \mathrm{N} / \mathrm{A}$ : not applicable.

\section{Additional Analyses}

\section{Distribution of Publications by Country}

Eighteen of the 46 studies (39\%) were performed in North America, 11 (24\%) were performed in Europe, 7 (15\%) were performed in Asia, 5 (11\%) were performed in Australia, $3(7 \%)$ were performed in South America, and $2(4 \%)$ were performed in multiple countries and continents.

\section{Comparisons to a Control Group}

Table 6 summarizes the themes and observations recorded for results as compared to the control group identified by the two reviewers. There is some overlap between this set of observations and medical outcomes; the latter represent clinical observations only, whereas the former are both clinical and administrative in nature. Ten themes and four individual observations were identified by the reviewers for a total of 66 occurrences in the literature. Eleven studies were nonexperimental in nature, which had no control group.
Eighteen of the studies demonstrated either a clinical or administrative improvement compared to the control group, whereas nine reported no statistically significant results from the control group. Both of these themes demonstrate the efficacy of the telemedicine modality. The remainder of the list in Table 6 demonstrates the specific improvements that occurred (multiple improvements occurred in multiple articles), including improved patient satisfaction, improved behaviors, improved compliance/adherence to treatment protocol, improved self-management of condition or disease, decreased fat mass, improved emotional support, improved companionship, and improved health literacy. The remainder were individual observations that combined accounted for $5 \%$ of the total observations: improved informational support, decreased cost, and increased quality of life. Only one article did not report a result as compared to the control group because it was a posttrial analysis and it did not address the control group. 
Table 6. Themes and individual observations for studies with a control group comparison ( $\mathrm{N}=66)$.

\begin{tabular}{lll}
\hline Themes/observations & References & Occurrence, $\mathrm{n}(\%)$ \\
\hline Telemedicine improved results compared to control & {$[28,32,35,38,40,41,43,46,48-50,53-55,58-60,64]$} & $18(27)$ \\
No statistically significant difference & {$[27,30,33,34,39,45,51,56,62]$} & $9(14)$ \\
Improved patient satisfaction & {$[24,25,43,47,49,50,63]$} & $7(11)$ \\
Improved health behaviors & {$[36,49,60,62,64,67]$} & $6(9)$ \\
Improved compliance/adherence & {$[22,38,40,41,49,67]$} & $6(9)$ \\
Improved self-management & {$[28,37,43,58,60]$} & $5(8)$ \\
Decreased fat mass & {$[27,32,35,58]$} & $4(6)$ \\
Improved emotional support & {$[28,55,63]$} & $3(5)$ \\
Improved companionship & {$[28,66]$} & $2(3)$ \\
Improved health literacy & {$[28,37]$} & $2(3)$ \\
Improved informational support & {$[28]$} & $1(2)$ \\
Decreased cost & {$[46]$} & $1(2)$ \\
Increased quality of life & {$[34]$} & $1(2)$ \\
Not reported & {$[57]$} & $1(2)$ \\
No control group (nonexperimental) & {$[23,26,29,31,37,41,42,44,52,61,65]$} & $11\left(\mathrm{~N} / \mathrm{A}^{\mathrm{a}}\right)$
\end{tabular}

${ }^{\mathrm{a} N} / \mathrm{A}$ : not applicable.

\section{Medical Outcomes Commensurate With an Intervention}

Table 7 summarizes the medical outcomes observed. Seven themes and nine individual observations were recorded commensurate with the adoption of telemedicine for a total of 30 occurrences. Twenty-eight studies did not report clinical outcomes.

Twelve studies reported 12 statistically significant improvements in clinical outcomes and three reported no statistically significant difference between modalities of care. Both of these themes demonstrated the efficacy of the telemedicine modality. The most commonly observed theme for medical outcomes was decreased body weight, followed by decreased fat mass, improved self-management, increase in controlled asthma, and increased quality of life. The following individual observations contributed to $30 \%$ of the total observations: reduction in blood pressure, reduction in mean tacrolimus trough coefficient of variation, improved annual rate of estimated glomerular filtration rate (eGFR) decline, decrease in medication use, decrease incidence of heart failure, decreased mortality, improved mental health inventory, decreased intracranial hemorrhage, and telemedicine identified other areas for intervention. 
Table 7. Medical outcome themes and individual observations commensurate with adoption of the intervention/technology $(\mathrm{N}=30)$.

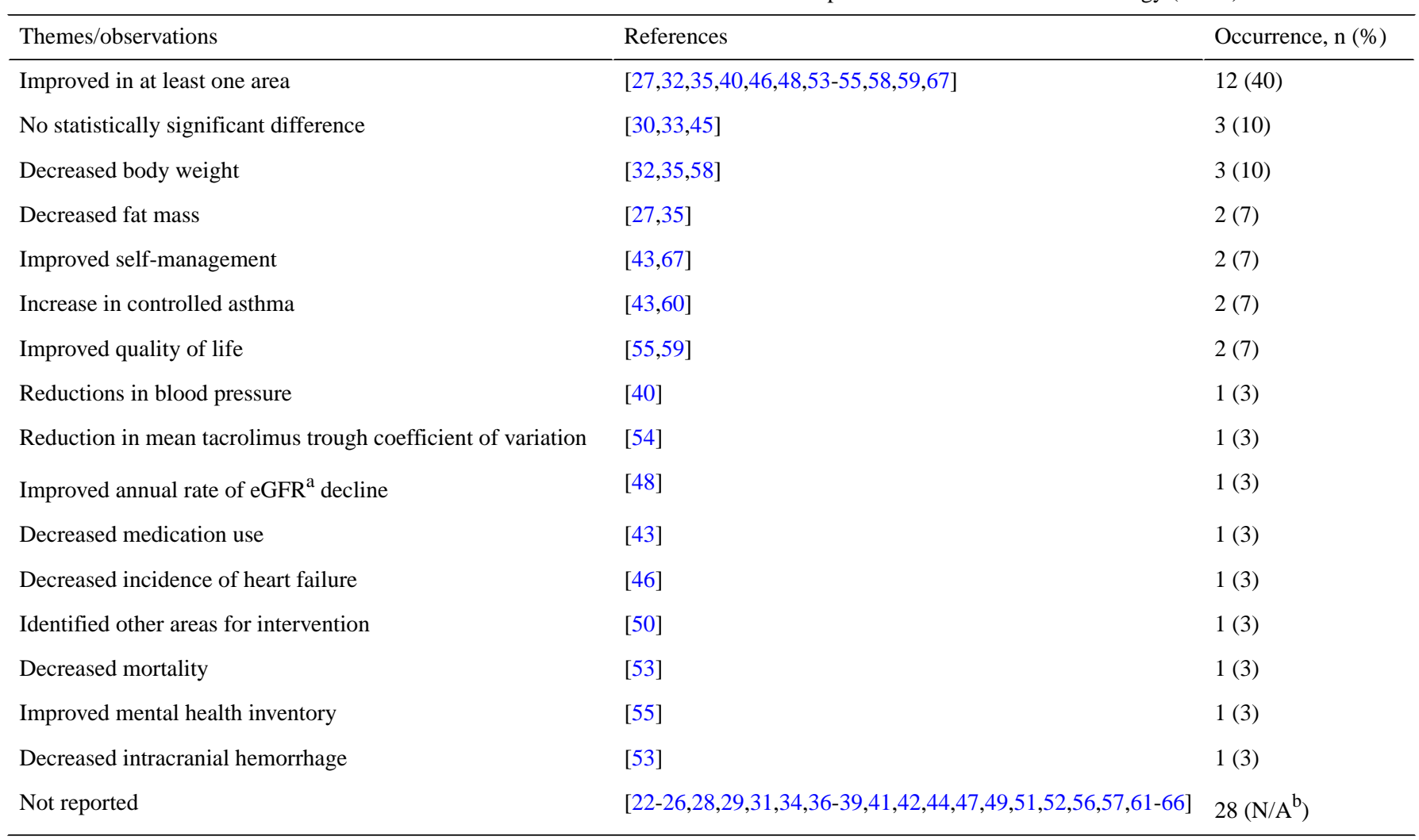

a eGFR: estimated glomerular filtration rate.

${ }^{b}$ N/A: not applicable.

\section{Interactions Between Observations}

Interventions of mHealth resulted in seven occurrences of a result (clinical and administrative outcomes) and six occurrences of an improvement in at least one clinical outcome. The interventions with telephone or televideo resulted in four instances of improved patient satisfaction and a decrease in eGFR and weight loss. The interventions of eHealth resulted in very few instances of either clinical or administrative outcomes other than improved compliance and health behaviors.

\section{Discussion}

\section{Principal Findings}

Telemedicine is examined in countries worldwide, and it is clear that the COVID-19 pandemic caused a rapid adoption of this modality of medicine to ensure the viability of practices. A key issue for discussion is the differences in findings between this systematic review and another recent similar review [14]. This systematic review identified key facilitators and barriers, and further analyzed health outcomes. The other similar review identified themes of effectiveness but failed to meet the expectations for a systematic review in terms of medical outcomes [68]. Common themes between the two reviews were: rapid telemedicine expansion, education, improved access, convenience, and patient satisfaction.

\section{Summary of Evidence}

This systematic review exercised a set Boolean search string in four common research databases to analyze 46 articles originating from five continents for themes of facilitators, barriers, and medical outcomes. Nearly $50 \%$ of the articles demonstrated the strongest evidence and nearly $60 \%$ demonstrated the highest quality of evidence. Various forms of telemedicine were examined: eHealth, mHealth, audio only, telemonitoring, telecoaching, telerehab, robotics or artificial intelligence, and televideo. Twenty-five facilitator themes and individual observations, 12 results themes and observations, and 7 medical outcome themes and observations were recorded and analyzed. Forty-one percent of barrier themes recorded either an improvement or no statistically significant improvement in results compared to the control group. Forty percent of the observations recorded an improvement in at least one medical outcome.

Health care administrators can focus on the findings demonstrating that implementation of telemedicine will increase self-management $\quad[27,30,35,37,38,40,41,45,46,49,57,58]$, adherence [32,34,35,37,38,40,43,46,58,67], access [24,27-30, $41,52,53,60]$, and social support [49,50,54,55,62-64,66]. Telemedicine is shown to be an effective modality of treatment $[28,32,35,38,40,41,43,46,48-50,53-55,58-60,64]$ at a decreased cost $[31,46,52,56,59,60]$. Patients perceive the modality to be convenient and easy to use [26,34,36,43-45,47], and its implementation increases patient satisfaction $[24,25,43$, 47,49,50,63].

Health policymakers should focus on several barriers to increase the adoption of telemedicine. Because technical literacy, availability of technology, and connectivity are listed as the most often cited barriers, public programs should be offered to 
assist those with these difficulties. Technical literacy is often associated along age or socioeconomic lines, and researchers acknowledge the dearth of research in the area of how to overcome this obstacle [69]. However, community centers that provide access to computers, classes on computers, and a dedicated broadband connection can all contribute to solutions to these barriers.

A key similarity between the 2020 systematic review [14] and this review is the rapid expansion of telemedicine. Eleven articles analyzed in this review used a phrase similar to "the pandemic created an acceptance of telemedicine technology" $[23,25,31,33,41,43,47,51,52,61,62]$. A systematic review published in 2018 cited cost as the chief barrier to adoption, whereas this review only found cost as a barrier in $8 \%$ of all observations [5]. The COVID-19 pandemic forced acceptance of the technology and enabled providers to not focus so intently on the cost of its implementation.

\section{Limitations}

This systematic review selected 46 articles for analysis from four commonly available research databases. A larger group for analysis could have yielded richer results. This review also only utilized two researchers to analyze the data; additional researchers could have identified additional themes. Selection bias was controlled through independent analysis of all articles by both reviewers followed by consensus meetings. Publication bias is the largest limitation because we were unable to query and analyze unpublished articles.

\section{Conclusion}

The COVID-19 pandemic caused huge problems to deliver medicine traditionally. However, these problems created an environment that limited face-to-face medical encounters and fostered legislation to reimburse the telemedicine modality for broad and rapid adoption of telemedicine to expand the access of care beyond the physical walls of the clinic. Physicians should feel confident that the telemedicine modality will be reimbursed and will have very little effect on patient satisfaction. Health care administrators who have not already adopted telemedicine should feel confident in the technology; however, they should ensure that sufficient confidentiality and security measures are in place. Policymakers should enact legislation to remove or mitigate barriers such as availability of technology, technical literacy, and connectivity, as these are commonly referred to in the literature.

\section{Authors' Contributions}

$\mathrm{KH}$ and CK contributed equally to this project. Both authors were involved in each step of the research and publication process.

\section{Conflicts of Interest}

None declared.

\section{Multimedia Appendix 1}

Sample size, bias, effect size, country of origin, and statistics used. [DOCX File, $60 \mathrm{~KB}-$ Multimedia Appendix 1]

\section{Multimedia Appendix 2}

Observation-to-theme conversion for patient satisfaction as well as facilitators and barriers to adoption. [DOCX File, $81 \mathrm{~KB}-$ Multimedia Appendix 2]

\section{References}

1. World Health Organization. Telemedicine: opportunities and developments in member states: report on the second global survey on eHealth 2009. Geneva, Swizerland: World Health Organization; 2010.

2. FitzGerald M, Williams R. Commonwealth Fund. 2020 Nov 20. URL: https://www.commonwealthfund.org/blog/2020/ variations-telemedicine-across-world-qa-tiago-cravo-oliveira-hashiguchi [accessed 2021-02-15]

3. Bokolo AJ. Exploring the adoption of telemedicine and virtual software for care of outpatients during and after COVID-19 pandemic. Ir J Med Sci 2021 Feb;190(1):1-10 [FREE Full text] [doi: 10.1007/s11845-020-02299-z] [Medline: 32642981]

4. Fieux M, Duret S, Bawazeer N, Denoix L, Zaouche S, Tringali S. Telemedicine for ENT: Effect on quality of care during Covid-19 pandemic. Eur Ann Otorhinolaryngol Head Neck Dis 2020 Sep;137(4):257-261 [FREE Full text] [doi: 10.1016/j.anorl.2020.06.014] [Medline: 32624390]

5. Kruse SC, Karem P, Shifflett K, Vegi L, Ravi K, Brooks M. Evaluating barriers to adopting telemedicine worldwide: A systematic review. J Telemed Telecare 2016 Oct 16;24(1):4-12. [doi: 10.1177/1357633x16674087]

6. Kuroda N. Decision making on telemedicine for patients with epilepsy during the coronavirus disease 2019 (COVID-19) crisis. Front Neurol 2020;11:722. [doi: 10.3389/fneur.2020.00722] [Medline: 32714276]

7. Ben-Pazi H, Beni-Adani L, Lamdan R. Accelerating telemedicine for cerebral palsy during the COVID-19 pandemic and beyond. Front Neurol 2020;11:746. [doi: 10.3389/fneur.2020.00746] [Medline: 32670193]

8. Royce TJ, Sanoff HK, Rewari A. Telemedicine for cancer care in the time of COVID-19. JAMA Oncol 2020 Nov 01;6(11):1698-1699. [doi: 10.1001/jamaoncol.2020.2684] [Medline: 32672821] 
9. Hong Z, Li N, Li D, Li J, Li B, Xiong W, et al. Telemedicine during the COVID-19 pandemic: experiences from Western China. J Med Internet Res 2020 May 08;22(5):e19577 [FREE Full text] [doi: 10.2196/19577] [Medline: 32349962]

10. Mojdehbakhsh RP, Rose S, Peterson M, Rice L, Spencer R. A quality improvement pathway to rapidly increase telemedicine services in a gynecologic oncology clinic during the COVID-19 pandemic with patient satisfaction scores and environmental impact. Gynecol Oncol Rep 2021 May;36:100708 [FREE Full text] [doi: 10.1016/j.gore.2021.100708] [Medline: 33521218]

11. Chang PJ, Jay GM, Kalpakjian C, Andrews C, Smith S. Patient and provider-reported satisfaction of cancer rehabilitation telemedicine visits during the COVID-19 pandemic. PM R 2021 Dec;13(12):1362-1368 [FREE Full text] [doi: 10.1002/pmrj.12552] [Medline: 33455066]

12. Duarte A, Gouveia E Melo R, Lopes A, Rato JP, Valente J, Pedro LM. Lessons learned from the impact of the COVID-19 pandemic in a vascular surgery department and preparation for future outbreaks. Ann Vasc Surg 2021 May;73:97-106 [FREE Full text] [doi: 10.1016/j.avsg.2021.01.060] [Medline: $\underline{\text { 33493593] }}$

13. Regelmann MO, Conroy R, Gourgari E, Gupta A, Guttmann-Bauman I, Heksch R, PES Drug and Therapeutics Committee and Practice Management Committee. Pediatric endocrinology in the time of COVID-19: considerations for the rapid implementation of telemedicine and management of pediatric endocrine conditions. Horm Res Paediatr 2020;93(6):343-350 [FREE Full text] [doi: 10.1159/000513060] [Medline: $\underline{\text { 33486483] }}$

14. Betancourt JA, Rosenberg MA, Zevallos A, Brown JR, Mileski M. The impact of COVID-19 on telemedicine utilization across multiple service lines in the United States. Healthcare (Basel) 2020 Oct 01;8(4):380 [FREE Full text] [doi: 10.3390/healthcare8040380] [Medline: 33019667]

15. Kruse CS. Writing a systematic review for publication in a health-related degree program. JMIR Res Protoc 2019 Oct 14;8(10):e15490 [FREE Full text] [doi: 10.2196/15490] [Medline: 31527018]

16. Page M, McKenzie J, Bossuyt P, Boutron I, Hoffmann T, Mulrow C, et al. The PRISMA 2020 statement: an updated guideline for reporting systematic reviews. BMJ 2021 Mar 29;372:n71 [FREE Full text] [doi: 10.1136/bmj.n71] [Medline: $\underline{33782057]}$

17. Newhouse R, Dearholt S, Poe S, Pugh L, White K. The Johns Hopkins nursing evidence-based practice rating scale. Johns Hopkins University School of Nursing. 2005. URL: https://www.google.com/url?sa=t\&rct=j\&q=\&esrc=s\&source=web\&cd =\&ved=2ahUKEwjuheqN3-30AhXummoFHa7zBSsQFnoECAMQAQ\&url=http\%3A\%2F\%2Fevidencebasednurse.weebly. com\%2Fuploads $\% 2 \mathrm{~F} 4 \% 2 \mathrm{~F} 2 \% 2 \mathrm{~F} 0 \% 2 \mathrm{~F} 8 \% 2 \mathrm{~F} 42081989 \% 2 \mathrm{Fjhnedp}$ evidence rating scale. pdf\&usg=AOvVaw0TqXuL2_eg1w4Da9XXoKNu [accessed 2021-02-15]

18. Light RJ. Measures of response agreement for qualitative data: Some generalizations and alternatives. Psychol Bull 1971;76(5):365-377. [doi: 10.1037/h0031643]

19. Braun V, Clarke V. Using thematic analysis in psychology. Qual Res Psychol 2006 Jan;3(2):77-101. [doi: 10.1191/1478088706qp063oa]

20. Moher D, Liberati A, Tetzlaff J, Altman D, PRISMA Group. Preferred reporting items for systematic reviews and meta-analyses: the PRISMA statement. PLoS Med 2009 Jul 21;6(7):e1000097 [FREE Full text] [doi: 10.1371/journal.pmed.1000097] [Medline: 19621072]

21. McHugh ML. Interrater reliability: the kappa statistic. Biochem Med 2012;22(3):276-282 [FREE Full text] [Medline: 23092060]

22. Ben-Arye E, Gressel O, Ben-Arye E, Samuels N. Feasibility of an online integrative oncology treatment program during COVID-19. J Pain Symptom Manage 2021 Feb;61(2):e1-e3 [FREE Full text] [doi: 10.1016/j.jpainsymman.2020.11.009] [Medline: 33189855]

23. Kurihara K, Nagaki K, Inoue K, Yamamoto S, Mishima T, Fujioka S, et al. Attitudes toward telemedicine of patients with Parkinson's disease during the COVID - 19 pandemic. Neurol Clin Neurosci 2020 Nov 18;9(1):77-82. [doi: 10.1111/ncn3.12465]

24. Richards AE, Curley K, Christel L, Zhang N, Kouloumberis P, Kalani MA, et al. Patient satisfaction with telehealth in neurosurgery outpatient clinic during COVID-19 pandemic. Interdisciplin Neurosurg 2021 Mar;23:101017. [doi: 10.1016/j.inat.2020.101017]

25. Yu J, Afridi SM, Cozart AC, Isea L, Guan J. Evaluation and feedback for telehealth from patients and physicians during the early stage of COVID-19 pandemic period. Cureus 2021 Jan 11;13(1):e12633 [FREE Full text] [doi: 10.7759/cureus.12633] [Medline: $\underline{33585121]}$

26. Alkire (née Nasr) L, O'Connor GE, Myrden S, Köcher S. Patient experience in the digital age: An investigation into the effect of generational cohorts. J Retail Consum Serv 2020 Nov;57:102221. [doi: 10.1016/j.jretconser.2020.102221]

27. Ballin M, Hult A, Björk S, Lundberg E, Nordström P, Nordström A. Web-based exercise versus supervised exercise for decreasing visceral adipose tissue in older adults with central obesity: a randomized controlled trial. BMC Geriatr 2020 May 12;20(1):173 [FREE Full text] [doi: 10.1186/s12877-020-01577-w] [Medline: 32398024]

28. Banbury A, Nancarrow S, Dart J, Gray L, Dodson S, Osborne R, et al. Adding value to remote monitoring: Co-design of a health literacy intervention for older people with chronic disease delivered by telehealth - The telehealth literacy project. Patient Educ Couns 2020 Mar;103(3):597-606. [doi: 10.1016/j.pec.2019.10.005] [Medline: $\underline{31744701]}$ 
29. Barnett A, Savic M, Pienaar K, Carter A, Warren N, Sandral E, et al. Enacting 'more-than-human' care: Clients' and counsellors' views on the multiple affordances of chatbots in alcohol and other drug counselling. Int J Drug Policy 2021 Aug;94:102910 [FREE Full text] [doi: 10.1016/j.drugpo.2020.102910] [Medline: 33059955]

30. Batalik L, Dosbaba F, Hartman M, Batalikova K, Spinar J. Benefits and effectiveness of using a wrist heart rate monitor as a telerehabilitation device in cardiac patients: A randomized controlled trial. Medicine (Baltimore) 2020 Mar;99(11):e19556. [doi: 10.1097/MD.0000000000019556] [Medline: 32176113]

31. Beller HL, Rapp DE, Noona SW, Winkelman AJ, Zillioux JM, Smith RP, et al. Tele-urology during COVID-19: rapid implementation of remote video visits. Urol Pract 2020 Nov;7(6):442-447. [doi: 10.1097/upj.0000000000000167]

32. Bernabe-Ortiz A, Pauschardt J, Diez-Canseco F, Miranda JJ. Sustainability of mHealth effects on cardiometabolic risk factors: five-year results of a randomized clinical trial. J Med Internet Res 2020 Apr 21;22(4):e14595 [FREE Full text] [doi: 10.2196/14595] [Medline: 32314970]

33. Bilgrami Z, Abutaleb A, Chudy-Onwugaje K, Langenberg P, Regueiro M, Schwartz DA, et al. Effect of TELEmedicine for inflammatory bowel disease on patient activation and self-efficacy. Dig Dis Sci 2020 Jan;65(1):96-103 [FREE Full text] [doi: 10.1007/s10620-018-5433-5] [Medline: $\underline{\text { 30604373] }}$

34. Broers ER, Kop WJ, Denollet J, Widdershoven J, Wetzels M, Ayoola I, et al. A personalized eHealth intervention for lifestyle changes in patients with cardiovascular disease: randomized controlled trial. J Med Internet Res 2020 May 22;22(5):e14570 [FREE Full text] [doi: 10.2196/14570] [Medline: 32441658]

35. Cho SMJ, Lee JH, Shim J, Yeom H, Lee SJ, Jeon YW, et al. Effect of smartphone-based lifestyle coaching app on community-dwelling population with moderate metabolic abnormalities: randomized controlled trial. J Med Internet Res 2020 Oct 09;22(10):e17435 [FREE Full text] [doi: 10.2196/17435] [Medline: 33034564]

36. Claes J, Cornelissen V, McDermott C, Moyna N, Pattyn N, Cornelis N, et al. Feasibility, acceptability, and clinical effectiveness of a technology-enabled cardiac rehabilitation platform (Physical Activity Toward Health-I): randomized controlled trial. J Med Internet Res 2020 Feb 04;22(2):e14221 [FREE Full text] [doi: 10.2196/14221] [Medline: 32014842]

37. Coorey G, Peiris D, Neubeck L, Redfern J. A realist evaluation approach to explaining the role of context in the impact of a complex eHealth intervention for improving prevention of cardiovascular disease. BMC Health Serv Res 2020 Aug 18;20(1):764 [FREE Full text] [doi: 10.1186/s12913-020-05597-5] [Medline: 32811480]

38. Ding H, Jayasena R, Chen SH, Maiorana A, Dowling A, Layland J, et al. The effects of telemonitoring on patient compliance with self-management recommendations and outcomes of the innovative telemonitoring enhanced care program for chronic heart failure: randomized controlled trial. J Med Internet Res 2020 Jul 08;22(7):e17559 [FREE Full text] [doi: 10.2196/17559] [Medline: 32673222]

39. Geramita E, DeVito Dabbs AJ, DiMartini A, Pilewski J, Switzer G, Posluszny D, et al. Impact of a mobile health intervention on long-term nonadherence after lung transplantation: follow-up after a randomized controlled trial. Transplantation 2020 Mar;104(3):640-651 [FREE Full text] [doi: 10.1097/TP.0000000000002872] [Medline: 31335759]

40. Gong K, Yan Y, Li Y, Du J, Wang J, Han Y, APP Study Group et al. Mobile health applications for the management of primary hypertension: A multicenter, randomized, controlled trial. Medicine (Baltimore) 2020 Apr;99(16):e19715. [doi: 10.1097/MD.0000000000019715] [Medline: 32311957]

41. Han JK, Al-Khatib SM, Albert CM. Changes in the digital health landscape in cardiac electrophysiology: a pre-and peri-pandemic COVID-19 era survey. Cardiovasc Dig Health J 2021 Feb;2(1):55-62. [doi: 10.1016/j.cvdhj.2020.12.001]

42. Harding R, Carrasco JM, Serrano-Pons J, Lemaire J, Namisango E, Luyirika E, et al. Design and evaluation of a novel mobile phone application to improve palliative home-care in resource-limited settings. J Pain Symptom Manage 2021 Jul;62(1):1-9. [doi: 10.1016/j.jpainsymman.2020.09.045] [Medline: 33246073]

43. Hsia BC, Singh AK, Njeze O, Cosar E, Mowrey WB, Feldman J, et al. Developing and evaluating ASTHMAXcel adventures: a novel gamified mobile application for pediatric patients with asthma. Ann Allergy Asthma Immunol 2020 Nov;125(5):581-588 [FREE Full text] [doi: 10.1016/j.anai.2020.07.018] [Medline: 32711031]

44. Hsieh P, Lai H. Exploring people's intentions to use the health passbook in self-management: An extension of the technology acceptance and health behavior theoretical perspectives in health literacy. Technol Forecast Soc Change 2020 Dec;161:120328. [doi: 10.1016/j.techfore.2020.120328]

45. Hutchesson MJ, Taylor R, Shrewsbury VA, Vincze L, Campbell LE, Callister R, et al. Be Health for Your Heart: a pilot randomized controlled trial evaluating a web-based behavioral intervention to improve the cardiovascular health of women with a history of preeclampsia. Int J Environ Res Public Health 2020 Aug 10;17(16):5779 [FREE Full text] [doi: 10.3390/ijerph17165779] [Medline: 32785044]

46. Jiménez-Marrero S, Yun S, Cainzos-Achirica M, Enjuanes C, Garay A, Farre N, et al. Impact of telemedicine on the clinical outcomes and healthcare costs of patients with chronic heart failure and mid-range or preserved ejection fraction managed in a multidisciplinary chronic heart failure programme: A sub-analysis of the iCOR randomized trial. J Telemed Telecare 2018 Sep 07;26(1-2):64-72. [doi: 10.1177/1357633x18796439]

47. Katt BM, Imbergamo C, Fletcher D, Aita D, Nakashian M, Kwok M, et al. Telehealth for upper extremity conditions: perceptions of the patient and provider. JAAOS Glob Res Rev 2020 Sep 10;4(9):e20.00127-e20.00113. [doi: 10.5435/jaaosglobal-d-20-00127] 
48. Kobe E, Diamantidis C, Bosworth H, Davenport C, Oakes M, Alexopoulos AS, et al. Racial differences in the effectiveness of a multifactorial telehealth intervention to slow diabetic kidney disease. Med Care 2020 Nov;58(11):968-973 [FREE Full text] [doi: 10.1097/MLR.0000000000001387] [Medline: 32833935]

49. Lai B, Bond K, Kim Y, Barstow B, Jovanov E, Bickel CS. Exploring the uptake and implementation of tele-monitored home-exercise programmes in adults with Parkinson's disease: A mixed-methods pilot study. J Telemed Telecare 2018 Aug 22;26(1-2):53-63. [doi: 10.1177/1357633x18794315]

50. Lemelin A, Paré G, Bernard S, Godbout A. Demonstrated cost-effectiveness of a telehomecare program for gestational diabetes mellitus management. Diabetes Technol Ther 2020 Mar;22(3):195-202. [doi: 10.1089/dia.2019.0259] [Medline: 31603351]

51. Manning B, Harpole A, Harriott E, Postolowicz K, Norton E. Taking language samples home: feasibility, reliability, and validity of child language samples conducted remotely with video chat versus in-person. J Speech Lang Hear Res 2020 Dec 14;63(12):3982-3990 [FREE Full text] [doi: 10.1044/2020 JSLHR-20-00202] [Medline: 33186507]

52. Marques G, Drissi N, Díez de la Torre I, de Abajo BS, Ouhbi S. Impact of COVID-19 on the psychological health of university students in Spain and their attitudes toward Mobile mental health solutions. Int J Med Inform 2021 Mar;147:104369. [doi: 10.1016/j.ijmedinf.2020.104369] [Medline: 33388481]

53. Martins SC, Weiss G, Almeida AG, Brondani R, Carbonera LA, de Souza AC, et al. Validation of a smartphone application in the evaluation and treatment of acute stroke in a comprehensive stroke center. Stroke 2020 Jan;51(1):240-246. [doi: 10.1161/strokeaha.119.026727]

54. McGillicuddy JW, Chandler JL, Sox LR, Taber DJ. Exploratory analysis of the impact of an mHealth medication adherence intervention on tacrolimus trough concentration variability: post hoc results of a randomized controlled trial. Ann Pharmacother 2020 Dec;54(12):1185-1193 [FREE Full text] [doi: 10.1177/1060028020931806] [Medline: $\underline{32506922]}$

55. Mo Y, Wang H, Huang G, Chu M. Effectiveness of nurse-led program on mental health status and quality of life in patients with chronic heart failure. Medicine (Baltimore) 2020 Aug 14;99(33):e21746. [doi: 10.1097/MD.0000000000021746] [Medline: 32872064]

56. Mustonen E, Hörhammer I, Absetz P, Patja K, Lammintakanen J, Talja M, et al. Eight-year post-trial follow-up of health care and long-term care costs of tele-based health coaching. Health Serv Res 2020 Apr;55(2):211-217 [FREE Full text] [doi: 10.1111/1475-6773.13251] [Medline: 31884682]

57. O'Shea O, Woods C, McDermott L, Buys R, Cornelis N, Claes J, et al. A qualitative exploration of cardiovascular disease patients' views and experiences with an eHealth cardiac rehabilitation intervention: The PATHway Project. PLoS One 2020;15(7):e0235274 [FREE Full text] [doi: 10.1371/journal.pone.0235274] [Medline: 32628688]

58. Perri MG, Shankar MN, Daniels MJ, Durning PE, Ross KM, Limacher MC, et al. Effect of telehealth extended care for maintenance of weight loss in rural US communities: a randomized clinical trial. JAMA Netw Open 2020 Jun 01;3(6):e206764 [FREE Full text] [doi: 10.1001/jamanetworkopen.2020.6764] [Medline: 32539150]

59. Piera-Jiménez J, Winters M, Broers E, Valero-Bover D, Habibovic M, Widdershoven JWMG, et al. Changing the health behavior of patients with cardiovascular disease through an electronic health intervention in three different countries: cost-effectiveness study in the Do Cardiac Health: advanced new generation ecosystem (Do CHANGE) 2 randomized controlled trial. J Med Internet Res 2020 Jul 28;22(7):e17351 [FREE Full text] [doi: 10.2196/17351] [Medline: 32720908]

60. Press VG, Arora VM, Kelly CA, Carey KA, White SR, Wan W. Effectiveness of virtual vs in-person inhaler education for hospitalized patients with obstructive lung disease: a randomized clinical trial. JAMA Netw Open 2020 Jan 03;3(1):e1918205 [FREE Full text] [doi: 10.1001/jamanetworkopen.2019.18205] [Medline: 31899529]

61. Ramírez-Correa P, Ramírez-Rivas C, Alfaro-Pérez J, Melo-Mariano A. Telemedicine acceptance during the COVID-19 pandemic: an empirical example of robust consistent partial least squares path modeling. Symmetry 2020 Sep 25;12(10):1593. [doi: 10.3390/sym12101593]

62. Ronan P, Mian A, Carr SB, Madge SL, Lorenc A, Robinson N. Learning to breathe with Tai Chi online - qualitative data from a randomized controlled feasibility study of patients with cystic fibrosis. Eur J Integr Med 2020 Dec;40:101229 [FREE Full text] [doi: 10.1016/j.eujim.2020.101229] [Medline: 33106755]

63. Sacco G, Lléonart S, Simon R, Noublanche F, Annweiler C, TOVID Study Group. Communication technology preferences of hospitalized and institutionalized frail older adults during COVID-19 confinement: cross-sectional survey study. JMIR Mhealth Uhealth 2020 Sep 18;8(9):e21845 [FREE Full text] [doi: 10.2196/21845] [Medline: 32896832]

64. Scheerman JFM, Hamilton K, Sharif MO, Lindmark U, Pakpour AH. A theory-based intervention delivered by an online social media platform to promote oral health among Iranian adolescents: a cluster randomized controlled trial. Psychol Health 2020 Apr;35(4):449-466. [doi: 10.1080/08870446.2019.1673895] [Medline: 31621423]

65. Schrauben S, Appel L, Rivera E, Lora C, Lash J, Chen J, CRIC Study Investigators. Mobile health (mHealth) technology: assessment of availability, acceptability, and use in CKD. Am J Kidney Dis 2021 Jun;77(6):941-950.e1. [doi: 10.1053/j.ajkd.2020.10.013] [Medline: 33309860]

66. Shareef MA, Kumar V, Dwivedi YK, Kumar U, Akram MS, Raman R. A new health care system enabled by machine intelligence: Elderly people's trust or losing self control. Technol Forecast Soc Change 2021 Jan;162:120334. [doi: 10.1016/j.techfore.2020.120334] 
67. van Dijk MR, Koster MPH, Oostingh EC, Willemsen SP, Steegers EAP, Steegers-Theunissen RPM. A mobile app lifestyle intervention to improve healthy nutrition in women before and during early pregnancy: single-center randomized controlled trial. J Med Internet Res 2020 May 15;22(5):e15773 [FREE Full text] [doi: 10.2196/15773] [Medline: $\underline{32412417]}$

68. Munn Z, Peters MDJ, Stern C, Tufanaru C, McArthur A, Aromataris E. Systematic review or scoping review? Guidance for authors when choosing between a systematic or scoping review approach. BMC Med Res Methodol 2018 Nov 19;18(1):143 [FREE Full text] [doi: 10.1186/s12874-018-0611-x] [Medline: 30453902]

69. Neumeyer X, Santos SC, Morris MH. Overcoming barriers to technology adoption when fostering entrepreneurship among the poor: the role of technology and digital literacy. IEEE Trans Eng Manage 2021 Dec;68(6):1605-1618. [doi: 10.1109/TEM.2020.2989740]

\author{
Abbreviations \\ CARES: Coronavirus Aid, Relief, and Economic Security \\ eGFR: estimated glomerular filtration rate \\ JHNEBP: Johns Hopkins Nursing Evidence-Based Practice Rating Scale \\ MeSH: Medical Subject Headings \\ mHealth: mobile health \\ PICOS: participants, intervention, results, outcomes, study design \\ PRISMA: Preferred Reporting Items for Systematic Reviews and Meta-Analyses
}

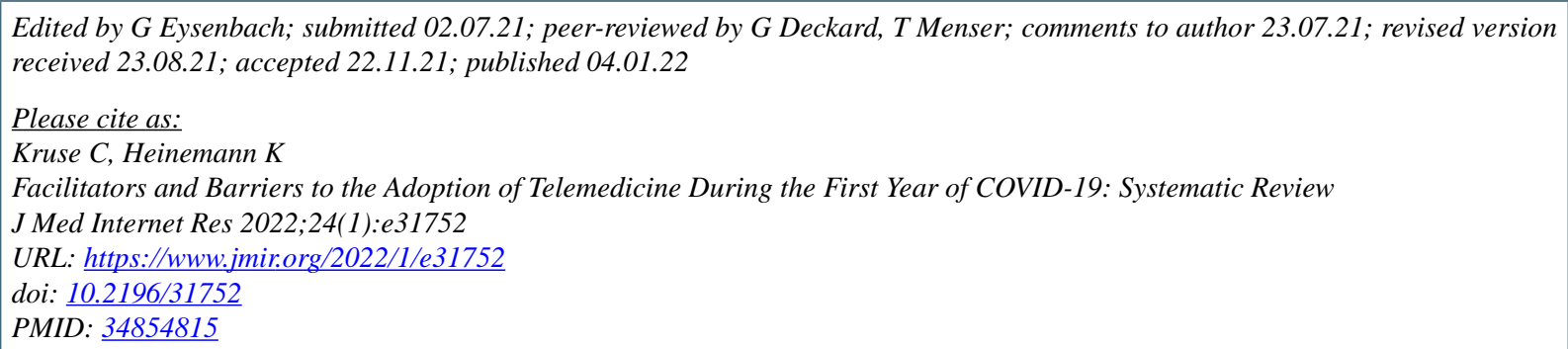

(C) Clemens Kruse, Katharine Heinemann. Originally published in the Journal of Medical Internet Research (https://www.jmir.org), 04.01.2022. This is an open-access article distributed under the terms of the Creative Commons Attribution License (https://creativecommons.org/licenses/by/4.0/), which permits unrestricted use, distribution, and reproduction in any medium, provided the original work, first published in the Journal of Medical Internet Research, is properly cited. The complete bibliographic information, a link to the original publication on https://www.jmir.org/, as well as this copyright and license information must be included. 